\title{
Laparoscopic sentinel node navigation surgery for early gastric cancer
}

\author{
Shinichi Kinami, Takeo Kosaka \\ Department of Surgical Oncology, Kanazawa Medical University, Kahoku, Japan \\ Contributions: (I) Conception and design: S Kinami; (II) Administrative support: T Kosaka; (III) Provision of study materials or patients: S Kinami; (IV) \\ Collection and assembly of data: S Kinami; (V) Data analysis and interpretation: S Kinami; (VI) Manuscript writing: All authors; (VII) Final approval \\ of manuscript: All authors. \\ Correspondence to: Shinichi Kinami. Department of Surgical Oncology, Kanazawa Medical University, 1-1 Daigaku, Uchinada-machi, Kahoku-gun, \\ Ishikawa 920-0293, Japan. Email: kinami@kanazawa-med.ac.jp.
}

\begin{abstract}
Currently, the most accurate method for identifying lymph node metastasis is intraoperative diagnosis by sentinel node (SN) biopsy. Based on the SNNS study - a recent large-scale, nationwide, multicenter prospective study-the SN concept seems to be scientifically valid in patients with early gastric cancer. SN biopsy is a multistep technique consisted of six essential elements: indication, the selection of a tracer, a proper tracer injection method, the objective detection of SNs, a reliable biopsy technique, and the precise detection of nodal metastasis. For SN biopsy of gastric cancer, these elements have been validated as follows: the indication should be limited to clinical T1 less than $4 \mathrm{~cm}$ in diameter; combination mapping with radioactive colloid and blue dye is used as the standard; and endoscopic submucosal injection is the standard tracer injection. Detection of SNs and a reliable biopsy technique are enabled by adaptation of lymphatic basin dissection, a proper biopsy technique for gastric cancer. Lymphatic basin dissection is a selective lymphadenectomy procedure for dissecting basins en bloc, collecting lymph nodes and lymphatic vessels stained with dye. Lymphatic basin dissection is superior to the ordinary pick-up method, not only for minimizing the rate of missed SNs, but also in terms of oncological safety as it complements an intraoperative frozen section diagnosis by serving as a backup dissection. Moreover, indocyanine green (ICG) fluorescence mapping has been developed in recent years. ICG fluorescence mapping is superior because of its high sensitivity and signal stability. Moreover, it is feasible for both open and laparoscopic gastrectomy in treating early gastric cancer. SN biopsy has brought dramatic changes to laparoscopic surgery for early gastric cancer. With laparoscopic SN biopsy using ICG fluorescence navigation, laparoscopic surgery for early gastric cancer has changed from the uniform standard gastrectomy with D1+ into a tailor-made function-preserving surgical procedure, such as local resection with lymphatic basin dissection.
\end{abstract}

Keywords: Sentinel nodes (SNs); gastric cancer; ICG fluorescence mapping; local resection

Received: 30 January 2017; Accepted: 07 April 2017; Published: 09 May 2017.

doi: $10.21037 / \operatorname{tgh} .2017 .05 .02$

View this article at: http://dx.doi.org/10.21037/tgh.2017.05.02

\section{Introduction}

A sentinel node ( $\mathrm{SN}$ ) is defined as the node that directly receives lymphatic drainage from a primary tumor (1). Therefore, the $\mathrm{SN}$ is thought to be the first node affected by lymph node micrometastasis. Hence detection and biopsy of $\mathrm{SN}$ are thought to be important for timely diagnosis and treatment of various cancers. In this chapter, we review the current advances in (and prospects of) SN biopsy for early gastric cancer.

\section{Quality of life (QoL) after laparoscopic gastrectomy}

Currently, laparoscopic gastrectomy is the most commonly 
used approach for early gastric cancers in East Asian countries such as China, Japan, and South Korea. Laparoscopic gastrectomy is less invasive and requires a smaller incision than ordinary open gastrectomy, however, it is more costly, requires a longer operation time, and is technically more difficult. Therefore, extensive efforts to overcome the difficulty of performing lymph node dissection have been made (2-4). It is difficult to perform precise lymph node dissection during open gastrectomy, with laparoscopic nodal dissection being even more difficult. Consequently, progress in laparoscopic gastrectomy has mainly focused on lymph node dissection techniques, and reproducing open curative gastrectomy. Laparoscopic gastrectomy merely replicates open standard gastrectomy. During these efforts to improve dissection, QoL after gastrectomy has not received as much attention as have the techniques themselves.

QoL after laparoscopic gastrectomy has not yet been sufficiently examined, largely because of a lack of an optimal tool for estimating postgastrectomy symptoms and QoL after gastrectomy. QoL is defined as a subjective assessment of an individual's emotional and physical well-being. In measuring and assessing of QoL, it is recommended that clinicians use a certain assessment tool that has been verified and certified under certain psychometric and healthcare approaches.

Until now, unlike the widespread assessment of QoL for cancer patients, there has been no optimal tool for assessing QoL after gastrectomy. Nakada et al. developed an integrated questionnaire, the Postgastrectomy Syndrome Assessment Scale (PGSAS)-45 (5). The PGSAS-45 was designed to assess living status and QoL in postgastrectomy patients. In using the PGSAS-45, researchers are able to scientifically examine the effect of laparoscopic gastrectomy on QoL. Accordingly, several studies have used the PGSAS-45 to assess QoL after laparoscopic gastrectomy. Notably, the QoL of patients after laparoscopic gastrectomy after 1 year is similar to that of patients after open standard gastrectomy (5-8).

For improvement of QoL after laparoscopic gastrectomy, it might be necessary to adopt function-preserving gastrectomy laparoscopically.

\section{The optimal laparoscopic function-preserving gastrectomy}

Function-preserving gastrectomy is a type of limited surgery used to treat early gastric cancer. It not only omits the extent of lymph node dissection, but it also maintains some stomach functions that are usually lost during standard gastrectomy. Preserved structures include the celiac, hepatic, and pyloric branches of the vagal nerve; the hepatic plexus; pylorus; and proximal side of stomach. There are various types of function-preserving gastrectomy, including proximal gastrectomy (7), pylorus-preserving gastrectomy (9), minimal distal gastrectomy, segmental gastrectomy $(10,11)$, and local (wedge) resection (12).

Standard lymph node dissection has been shown to improve survival in patients with gastric cancer $(13,14)$. Therefore, after the development of endoscopic submucosal dissection (ESD), curative surgery for gastric cancer now demands not only resection of the cancer, but also prophylactic dissection of the regional lymph nodes. Of course, the goal of cancer surgery is to cure cancer. The preservation of autonomic nerves may compromise the curability of lymph node dissection. The preservation of specific parts of the stomach also demands a continual blood supply achieved by the omission of part of prophylactic lymph node dissection. Therefore, the indications for function-preserving gastrectomies must be carefully considered. Furthermore, in contrast to breast cancer, additional lymph node dissection is rarely performed for gastric cancers.

As previously discussed, regarding operation time and technical difficulty, laparoscopic surgery is inferior to open surgery. In addition, the assurance of curability of functionpreserving gastrectomy is unproven. Considering together, laparoscopic function-preserving gastrectomy therefore should be planned carefully to accomplish a good functional outcome and to facilitate technical feasibility. Among the procedures associated with function-preserving gastrectomy, minimal distal gastrectomy and segmental gastrectomy are unique. Few reports of these procedures exist, even in open surgery (10). Therefore, proximal gastrectomy, pylorus-preserving gastrectomy, and local resection are the potential choices for safe laparoscopic functional-preserving gastrectomy.

Previous articles have assessed QoL after functionpreserving gastrectomy using the PGSAS-45 scale. Studies of large cohorts using the PGSAS demonstrated that pylorus-preserving gastrectomy is superior to distal gastrectomy since it has a lower incidence of dumping symptoms (9). The indications for pylorus-preserving gastrectomy have been previously established by several researchers, and this procedure is largely reproducible using laparoscopic surgery. Nevertheless, the other functional 
outcomes were found to be similar between pyloruspreserving gastrectomy and distal gastrectomy (9), and it is still difficult to perform upright gastro-gastrostomy of pylorus-preserving gastrectomy without an upper abdominal incision $(15,16)$.

Studies using the PGSAS also demonstrated that proximal gastrectomy is superior to total gastrectomy in terms of body weight loss, prevention of diarrhea, and lower incidence of dumping symptoms (7). However, some problems remain regarding the appropriate indications for proximal gastrectomy, and it is difficult to easily create antireflux esophagogastrostomy during laparoscopic proximal gastrectomy (17). Thus, laparoscopic pylorus-preserving gastrectomy and laparoscopic proximal gastrectomy are still considered experimental procedures, although they are common in open surgery.

Therefore, the most promising approach for laparoscopic function-preserving gastrectomy is likely to be local resection of the stomach (12).

\section{Requirements of the laparoscopic local resection of stomach}

"Local resection of the stomach" refers to a surgical procedure involving full-thickness, resection of a part of the gastric wall (12); the term is synonymous with "wedge resection". The advantage of local resection is that it minimizes functional impairment. Local resection was shown by the PGSAS studies to be more superior to standard gastrectomy in terms of almost all subscales of the PGSAS $(5,6)$.

When conducting local resection, it is essential to maintain a sufficient blood supply. Hence a case without lymph node metastasis is the best indication for local resection. It is possible to predict node-negative cases by studying the clinicopathological features during the preoperative stage. At the end of the 20th Century, centers specializing in the treatment of gastric cancer performed local resections daily (12). A large number of laparoscopic local resections of the stomach were also performed worldwide. The lesion lifting method developed by Ohgami et al. (18) became popular due to its simplicity. However, the treatment strategy for these node-negative early gastric cancer cases changed dramatically after the development of endoscopic treatment. At present, almost all early gastric cancer cases suspected as being node-negative are considered for ESD, and thus the need for local resection of the stomach has decreased. Indeed, laparoscopic local resection of the stomach for gastric cancer is now rarely performed (12). However, among the cases of surgical resections for early gastric cancer, only $20 \%$ have nodal metastasis; therefore, nearly $80 \%$ of cases have undergone unnecessary nodal dissection, which is excessive. Such cases might be potent cases for local resection. Improved techniques for accurately identifying lymph node metastasis would increase the proportion of early gastric cancer cases in which unnecessary dissection is avoided, and more local resections of the stomach would result.

Intraoperative diagnosis by $\mathrm{SN}$ biopsy is the most accurate method of identifying lymph node metastasis. SN biopsy may be essential for conducting laparoscopic function-preserving gastrectomy and for improving the QoL of patients suffering from early gastric cancer (19-21).

\section{Feasibility of SN biopsy for early gastric cancer}

SN biopsy has two main purposes in various cancers: ultrastaging and guidance for omission of lymph node dissection. In malignant melanoma management, SN biopsy is primarily used for ultrastaging (1), whereas in breast cancer treatment it is mainly used to guide lymph node dissection omission during surgery (22). Gastric cancer has been thoroughly investigated by several researchers trying to determine the validity of the $\mathrm{SN}$ concept, to apply an omission of nodal dissection like breast cancer (19-21,23-26).

Over recent decades, SN mapping for gastric cancer has been controversial because lymphatic flow around the stomach is complex (27). However, in many recent prospective studies, the usefulness of SN biopsy for gastric cancer has been successfully demonstrated (20,23-27). Therefore, two, large-scale, nationwide, multicenter prospective studies were carried out to verify the usefulness of SN biopsy for gastric cancer in Japan: the JCOG0302 study (28) and the SNNS study (29). Unfortunately, the JCOG0302 study was terminated because of a high false negative rate in intraoperative histological examinations. Whereas, the sole purpose of the SNNS study was to scientifically verify the $\mathrm{SN}$ concept in gastric cancer, and the current study was designed to minimize the ability of intraoperative pathological diagnosis to affect the results. Participating institutions were limited to those with physicians skilled in SN biopsies and with experience in more than 30 cases. Permanent histological sections were used for diagnoses of metastases; diagnostic sensitivity of intraoperative frozen section was only $79 \%$. In this study, 
the sensitivity and specificity of SN biopsy were $93 \%$ and $99 \%$, respectively. Based on these findings, the effectiveness of the SN concept used in the treatment of small-sized early gastric cancers was demonstrated scientifically.

\section{Technical details of SN mapping for gastric cancer}

The SN biopsy is a complex, multistep, surgical technique. Indeed, it is well known that an extensive learning phase is required before clinicians can master SN biopsy. For breast cancer, clinicians usually must perform 40 cases to learn how to properly perform the SN biopsy (30). Similarly, it has been proposed that clinicians should perform 30 cases in order to learn the proper technique for SN biopsy of gastric cancer (29).

The SN biopsy consists of six essential elements: limited proper indication, the selection of an adequate tracer, an appropriate tracer injection method, the objective detection of nodes that have taken up the tracer, a reliable biopsy technique for the nodes, and the precise intraoperative detection (micrometastasis level) of nodal metastasis (31).

Careful selection of patients is important to obtain successful results for SN biopsy. There are two important points that determine whether a case is suitable for SN biopsy. First, clinically node-negative cases should be selected. Second, surgeons should consider the adequate size and depth of invasion. According to the SNNS study (29) and a meta-analysis of SN biopsy for gastric cancer conducted by Wang et al. (32), the indication should be limited to clinical $\mathrm{T} 1$ cancers less than $4 \mathrm{~cm}$ in diameter.

In detecting the $\mathrm{SN}$, it is common to use tracers that flow from the injection site surrounding the primary tumor to the $\mathrm{SN}$ through the primary drainage lymphatic canals. Indeed, it is important to use an adequate tracer for SN mapping. In the SNNS study (29), combination mapping with technetium-99m tin colloid and isosulfan blue was used, which has since been adopted as a standard (31). Dye methods have been most commonly used for SN biopsy in various cancer surgeries. One of the advantages of dye methods is the direct visualization of the lymphatic canals and primary lymphatic drainage areas. The other advantages of dye include their low cost, easy availability, tractability, and lack of radioactivity. The disadvantages of the dye method include lack of objectivity, difficulty in digitizing, quick wash out and deterioration, unusual allergic reactions, and (at times) difficulty in detecting nodes in obese patients. Radioactive imaging (RI) colloid tracers are also common in SN biopsy. Advantages of this method include the high detection ability of the SN, its ability to withstand time deterioration of tracer uptake, rare allergic reactions, objectivity, ease of digitizing, and possibility of easily distinguishing SN from secondary nodes. Conversely, the disadvantages of the RI method include its high cost, difficulty in handling, radioactivity, no visual detection, difficulty in detection of lymphatic vessels and lymphatic basin, and the shine-through effect in the surgical field. Combination mapping is thought to be superior to single mapping because of its synergistic effect, thereby allowing surgeons to detect both primary lymphatic canals visually and the SN objectively (33).

The selected method for the tracer injection is important as well as the choice of tracer. For gastric cancer, two injection methods are suitable: endoscopic submucosal injection (20) or intraoperative subserosal injection behind the tumor (23). The de facto standard procedure of tracer injection is now the endoscopic submucosal injection adopted in the SNNS study, without regard to the type of tracers or the location of the tumor. In Wang and colleagues' meta-analysis of SN biopsy for gastric cancer, the submucosal injection method was found to be associated with a higher identification rate and sensitivity (32).

\section{Lymphatic basin dissection-essential for SN biopsy for gastric cancer}

In contrast to the former three elements, the remaining three elements are technically difficult, which contributes to the need for sufficient training to accurately determine the results of SN biopsy. Among these issues, objective detection and reliable biopsy technique issues could be resolved by the adaptation of a proper biopsy technique for SN biopsy, namely lymphatic basin dissection $(20,21)$. The lymphatic basin is defined as the lymphatic zone divided by the stream of the dyed lymphatic canals in dye mapping. The proximal border of the lymphatic basin is the fatty tissue attached to the stomach wall, and the distal border of the basin is the front of the stained node furthest from the stomach. All SNs exist within the lymphatic basin. The lymphatic basins are thought to be the primary lymphatic drainage areas in each patient. Patients having gastric cancer often have two or three basins (21). Lymphatic basin dissection is a selective lymphadenectomy to dissect lymphatic basins en bloc (Figure 1). SNs are harvested after lymphatic basin dissection and are sent for intraoperative pathological analysis. Lymphatic basin dissection is superior 


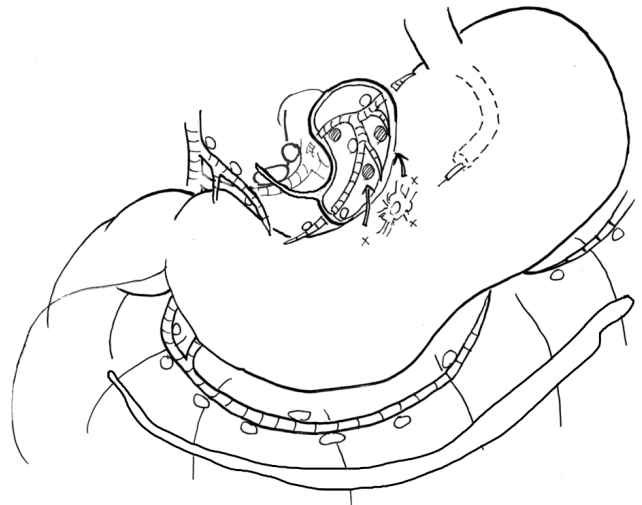

Figure 1 The schema of the lymphatic basin dissection. The laparoscopic dye-guided sentinel node mapping is performed during surgery. At first, the gastrocolic ligament is divided to visualize all lymphatic flows from the stomach. Next, blue dye tracer is injected via intraoperative endoscopy in four quadrants of the submucosal layer of the primary lesion by using an endoscopic needle. The lymphatic basin is defined as the zone divided by the stream of the dyed lymphatic canals in mapping; the proximal border is the fatty tissue attached to the stomach wall; and the distal border is the front of the blue node furthest from the stomach. The en bloc dissection of the lymphatic basin is performed laparoscopically.

to the ordinary pick-up method, not only for minimizing the risk of missed SNs, but also in terms of oncological safety as it complements intraoperative pathology by serving as a backup dissection (21).

At the SN biopsy for gastric cancer, the most difficult issue is intraoperative diagnosis of lymph node metastasis. Unlike breast cancer, re-operation for additional nodal dissection is not acceptable in the case of gastric cancer. Therefore, extremely accurate intraoperative detection of nodal metastasis (up to a micrometastasis level) is necessary. Normally, intraoperative rapid diagnosis of lymph node metastasis is performed by examining the largest dimension in one plane of the frozen section stained by rapid hematoxylin-eosin staining. Unfortunately, the JCOG0302 study was abandoned because of the higher false negative rate of intraoperative histological examinations (28). This failure was indicated by the fact that intraoperative histological examination using a single plane was not suitable for clinical applications.

To solve this difficult problem and to establish oncological safety, molecular diagnosis of nodal metastasis, such as reverse transcriptase-polymerase chain reaction (34) or one-step nucleic acid amplification assay (35), should be applied for clinical use. One practical solution is probably backup nodal dissection. In addition, lymphatic basin dissection is another possible solution to this problem. Kinami et al. reported that there were no recurrent cases in 190 patients diagnosed as node-negative by SN biopsy intraoperatively and who were treated by functionpreserving gastrectomy with lymphatic basin dissection (21).

\section{Technical difficulty and overcoming strategy for laparoscopic SN biopsy}

The QoL of patients suffering from gastric cancer after laparoscopic surgery might improve by adapting local resection guided by $\mathrm{SN}$ mapping and lymphatic basin dissection. However, two problems still exist that relate to the technical difficulty of laparoscopic surgery. One problem is related to the characteristics of the tracers (31).

Based on the result of the SNNS study, combination mapping with tin colloid and blue dye has been adopted as a temporary standard technique of SN biopsy for early gastric cancer. However, blue dye deteriorates quickly, and RI colloids exhibit a shine-through effect in the surgical field during gamma probe detection of hot nodes. Furthermore, the laparoscopic approach is time-consuming and its effectiveness is stymied by the angle-directional limitations of surgical devices.

Therefore, these weak characteristics of tracers limit their usefulness for laparoscopic SN biopsy. The possible best way to overcome this problem would be to improve the tracer. The ideal tracer for laparoscopic surgery is thought to have the following characteristics: easy to visualize the lymphatic canals, good permeability and strong accumulation in the $\mathrm{SN}$, and little flow out from the SN to secondary nodes. Visualization and less deterioration against time are especially important.

Indocyanine green (ICG) is a suitable tracer for laparoscopic SN biopsy. In recent years, ICG fluorescence SN mapping has been developed (36-38). ICG emits maximal fluorescence at a wavelength of $840 \mathrm{~nm}$ when it binds to plasma proteins. ICG fluorescence imaging was revived after the recent invention of the camera system known as the Photodynamic Eye (PDE; Hamamatsu Photonics Co. Ltd., Hamamatsu, Shizuoka, Japan). Kusano et al. demonstrated high sensitivity for this ICG fluorescence SN mapping in gastric cancer (36).

The advantages of ICG fluorescence imaging are lower frequency of allergic reactions than that with blue dye, 


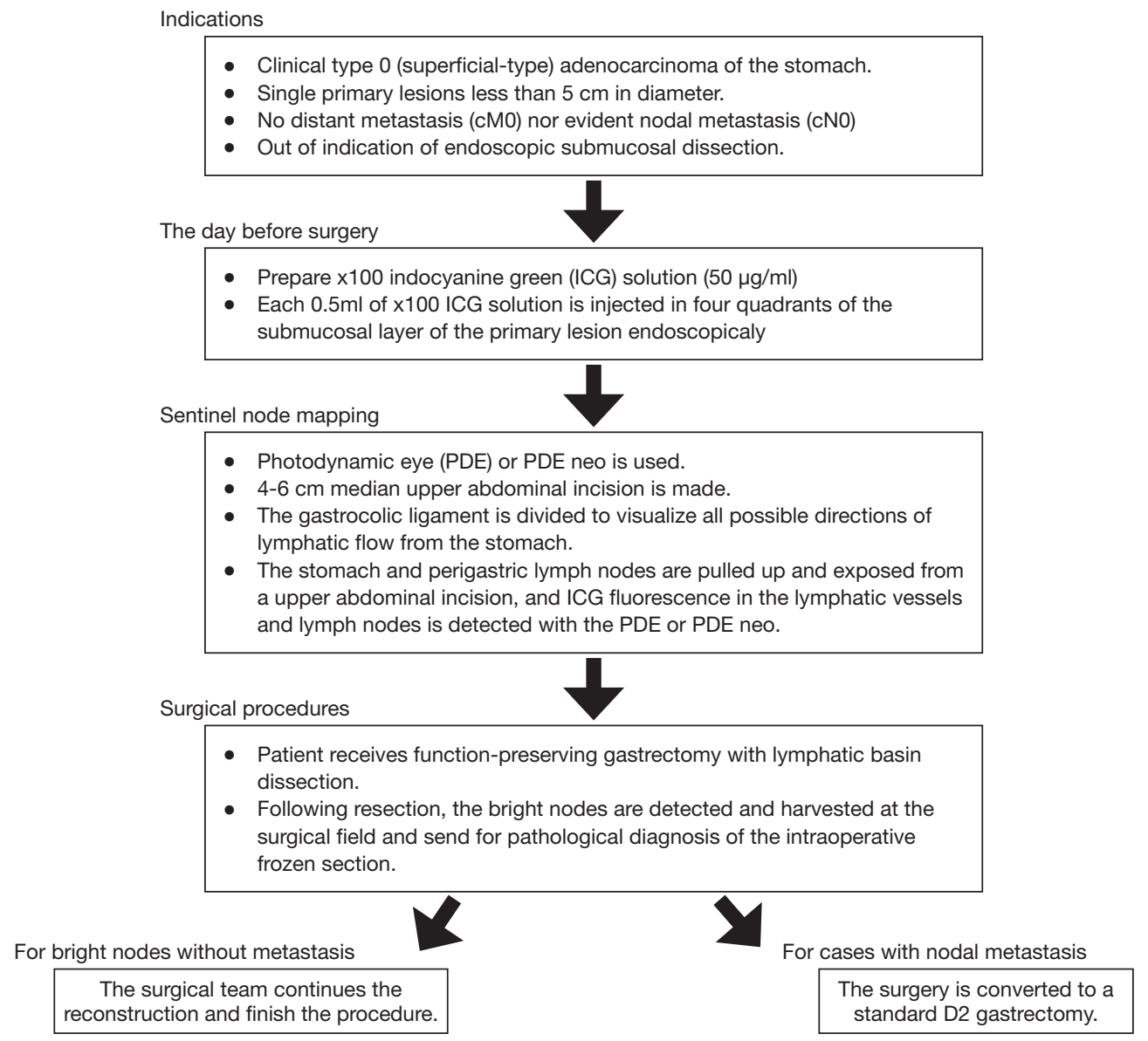

Figure 2 Diagram of the ICG fluorescence sentinel node mapping procedure for gastric cancer, which is suitable for laparoscopic surgery. $\mathrm{ICG}$, indocyanine green.

ability to detect bright nodes under adipose tissue, obvious visualization, easy detection of bright nodes and lymphatic canals, high sensitivity to detect minute concentrations of ICG, and signal stability (36-38). Signal stability is the most important advantage of ICG fluorescence SN mapping over dye or combination mapping (38). Kinami et al. carefully investigated ICG fluorescence SN mapping for gastric cancer (31). The authors concluded that ICG fluorescence SN biopsy is feasible in both open and laparoscopic surgery for early gastric cancer, and the optimal tracer setting to use with the PDE is an endoscopic submucosal injection of $0.5 \mathrm{~mL}$ of $50 \mu \mathrm{g} / \mathrm{mL}$ ICG at four points surrounding the tumor the day before surgery. Figure 2 shows a diagram of the ICG fluorescence SN mapping procedure for gastric cancer using PDE. Fortunately, several ICG fluorescence imaging systems have been developed, including the
IMAGE 1 HD system by Karl Storz, the IRI system by Olympus, the ICG fluorescent laparoscope by SinkoOptical, and the PINPOINT system by Novadaq. More use of these systems would enable widespread ICG fluorescence imaging and function-preserving gastrectomy (31).

Nevertheless, some disadvantages of ICG fluorescence imaging have been reported, and careful management for correct clinical application is required. Kinami et al. reported how weakness of ICG fluorescence mapping depends on the subjectivity of the SN evaluation, and potential secondary node contamination is possible because of the high sensitivity of the PDE system (31). However, these limitations will soon be overcome. Indeed, new agents have already been developed that have both fluorescence and colloid particle characteristics $(39,40)$. These new agents will be the most useful for conducting laparoscopic 

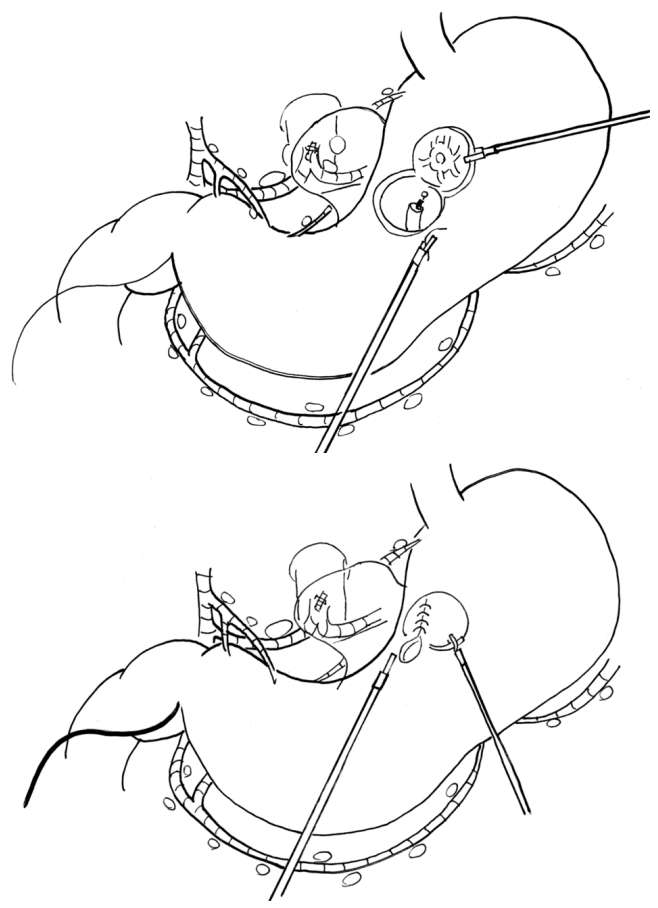

Figure 3 Schema of laparoscopic local resection guided by lymphatic basin dissection. These schemas are continuations of Figure 1. After being diagnosed as node negative by intraoperative pathological diagnosis, the combination of endoscopic fullthickness resection of the stomach using an ESD device and suturing closure using a laparoscope or robot is performed in establishing both the oncological safety and well preserved functions. ESD, endoscopic submucosal dissection.

SN biopsy in gastric cancer since they only detect fluorescent SNs and not secondary nodes.

\section{The future of laparoscopic surgery for early gastric cancer}

SN biopsy would bring dramatic change to laparoscopic surgery for early gastric cancer. Under guidance of laparoscopic SN biopsy using proper fluorescent tracer, the laparoscopic surgery for early gastric cancer would change from the uniform standard gastrectomy with D1+ into a tailor-made function-preserving surgery such as local resection with lymphatic basin dissection (12) (Figure 3). Although many problems remain to be solved before being able to properly perform laparoscopic SN mapping (such as diagnosis of micrometastasis, clinical application of proper fluorescent agent, and sensitivity of newly developed fluorescent laparoscopy), laparoscopic functionpreserving gastrectomy will likely be increasingly used since postgastrectomy symptoms are lifelong problems. Resolution of the discomfort caused by these symptoms is invaluable to many cancer survivors. Moreover, it is easy to conduct lymphatic basin dissection laparoscopically for surgeons who can perform D1+ laparoscopically.

Unfortunately there is another barrier to the widespread use of laparoscopic function-preserving gastrectomy: the technical difficulty of laparoscopic local resection itself (12). Laparoscopic local resection of the stomach is both an old and novel method. It was developed by Ohgami $e t a l$. as a lesionlifting method (18) following the development of laparoscopic linear stapler devices. Until now, there have been several approaches for laparoscopic local resection, such as the nontouch lesion lifting method (41), endoscopic full-thickness resection (EFTR) with laparoscopic closure (42), and laparoscopic endoscopic cooperative surgery (LECS) (43). The diseases treated with these techniques include small early gastric cancer under $2 \mathrm{~cm}$ in diameter (treated using the lesion lifting method), and gastrointestinal stromal tumors treated using other techniques.

In contrast to these small or submucosal solid tumors, the theoretical targets of laparoscopic local resection guided by SN biopsy included submucosal cancer tumors larger than $2 \mathrm{~cm}$. It is difficult to resect large areas of the stomach using the lesion-lifting method or the non-touch lesion lifting method because it is difficult to precisely resect a large area, and severe complications such as deformity may result. EFTR or LECS would be a suitable technique for such early gastric cancer because these techniques can create a precise minimum margin of about $5 \mathrm{~mm}$. Nevertheless, these techniques have a possible weak point: intraperitoneal spillage of the gastric contents.

It may be necessary during gastric cancer surgery to prevent intraperitoneal spillage of gastric contents and to minimize tumor exposure, because there have been reports of recurrence due to peritoneal metastasis in cases of early gastric cancer that was complicated by perforation occurring during ESD (44). In ordinary EFTR or LECS, the prevention of spillage of the gastric contents into the peritoneum is difficult. Reported methods of preventing intraperitoneal spillage of gastric contents-including inverted LECS (Crown method) (45), CLEAN-NET (46), NEWS (47), and sealed EFTR (48)—have advantages as well as limitations. Robotic surgery may provide the ideal solution to these difficulties. 


\section{Acknowledgements}

The author is most grateful to Dr. Koichi Miwa and Dr. Takashi Fujimura. They are top researchers of the sentinel node navigation surgery for gastric cancer, and mentors of Shinichi Kinami.

\section{Footnote}

Conflicts of Interest: The authors have no conflicts of interest to declare.

\section{References}

1. Morton DL, Wen DR, Wong JH, et al. Technical details of intraoperative lymphatic mapping for early stage melanoma. Arch Surg 1992;127:392-9.

2. Fukunaga T, Hiki N, Kubota T, et al. Oncologic outcomes of laparoscopy-assisted distal gastrectomy for gastric cancer. Ann Surg Oncol 2013;20:2676-82.

3. Katai H, Sasako M, Fukuda H, et al. Safety and feasibility of laparoscopy-assisted distal gastrectomy with suprapancreatic nodal dissection for clinical stage I gastric cancer: a multicenter phase II trial (JCOG 0703). Gastric Cancer 2010;13:238-44.

4. Inaki N, Etoh T, Ohyama T, et al. A Multi-institutional, Prospective, Phase II Feasibility Study of LaparoscopyAssisted Distal Gastrectomy with D2 Lymph Node Dissection for Locally Advanced Gastric Cancer (JLSSG0901). World J Surg 2015;39:2734-41.

5. Nakada K, Ikeda M, Takahashi M, et al. Characteristics and clinical relevance of postgastrectomy syndrome assessment scale (PGSAS)-45: newly developed integrated questionnaires for assessment of living status and quality of life in postgastrectomy patients. Gastric Cancer 2015;18:147-58.

6. Nakada K, Takahashi M, Ikeda M, et al. Factors affecting the quality of life of patients after gastrectomy as assessed using the newly developed PGSAS-45 scale: A nationwide multi-institutional study. World J Gastroenterol 2016;22:8978-90.

7. Takiguchi N, Takahashi M, Ikeda M, et al. Long-term quality-of-life comparison of total gastrectomy and proximal gastrectomy by postgastrectomy syndrome assessment scale (PGSAS-45): a nationwide multiinstitutional study. Gastric Cancer 2015;18:407-16.

8. Terashima M, Tanabe K, Yoshida M, et al. Postgastrectomy Syndrome Assessment Scale (PGSAS)-45 and changes in body weight are useful tools for evaluation of reconstruction methods following distal gastrectomy. Ann Surg Oncol 2014;21 Suppl 3:S370-S378.

9. Fujita J, Takahashi M, Urushihara T, et al. Assessment of postoperative quality of life following pyloruspreserving gastrectomy and Billroth-I distal gastrectomy in gastric cancer patients: results of the nationwide postgastrectomy syndrome assessment study. Gastric Cancer 2016;19:302-11.

10. Fujimura T, Fushida S, Kayahara M, et al. Transectional gastrectomy: an old but renewed concept for early gastric cancer. Surg Today 2010;40:398-403.

11. Shinohara T, Ohyama S, Muto T, et al. Clinical outcome of high segmental gastrectomy for early gastric cancer in the upper third of the stomach. Br J Surg 2006;93:975-80.

12. Kinami S, Funaki H, Fujita H, et al. Local resection of the stomach for gastric cancer. Surg Today 2017;47:651-9.

13. Songun I, Putter H, Kranenbarg E, et al. Surgical treatment of gastric cancer: 15-year follow-up results of the randomised nationwide Dutch D1D2 trial. Lancet Oncol 2010;11:439-49.

14. Degiuli M, Sasako M, Ponti A, et al. Randomized clinical trial comparing survival after D1 or D2 gastrectomy for gastric cancer. Br J Surg 2014;101:23-31.

15. Namikawa T, Hiki N, Kinami S, et al. Factors that minimize postgastrectomy symptoms following pylorus-preserving gastrectomy: assessment using a newly developed scale (PGSAS-45). Gastric Cancer 2015;18:397-406.

16. Koeda K, Chiba T, Noda H, et al. Intracorporeal reconstruction after laparoscopic pylorus-preserving gastrectomy for middle-third early gastric cancer: a hybrid technique using linear stapler and manual suturing. Langenbecks Arch Surg 2016;401:397-402.

17. Muraoka A, Kobayashi M, Kokudo Y. LaparoscopyAssisted Proximal Gastrectomy with the Hinged Double Flap Method. World J Surg 2016;40:2419-24.

18. Ohgami M, Otani Y, Kumai K, et al. Curative laparoscopic surgery for early gastric cancer: five years experience. World J Surg 1999;23:187-92.

19. Miwa K. Sentinel node concept and its application for cancer surgery. Nihon Geka Gakkai Zasshi 2000;101:307-10.

20. Miwa K, Kinami S, Taniguchi K, et al. Mapping sentinel nodes in patients with early-stage gastric carcinoma. $\mathrm{Br} \mathrm{J}$ Surg 2003;90:178-82.

21. Kinami S, Fujimura T, Ojima E, et al. PTD classification: proposal for a new classification of gastric cancer location 
based on physiological lymphatic flow. Int J Clin Oncol 2008;13:320-9.

22. Giuliano AE, Haigh PI, Brennan MB, et al. Prospective observational study of sentinel lymphadenectomy without further axillary dissection in patients with sentinel nodenegative breast cancer. J Clin Oncol 2000;18:2553-9.

23. Hiratsuka M, Miyashiro I, Ishikawa O, et al. Application of sentinel node biopsy to gastric cancer surgery. Surgery 2001;129: 335-40.

24. Carlini M, Carboni F, Petric M, et al. Sentinel node in gastric cancer surgery. J Exp Clin Cancer Res 2002;21:469-73.

25. Ichikura T, Morita D, Uchida T, et al. Sentinel node concept in gastric carcinoma. World J Surg 2002;26:318-22.

26. Kitagawa Y, Fujii H, Mukai M, et al. Radio-guided sentinel node detection for gastric cancer. Br J Surg 2002;89:604-8.

27. Maruyama K, Sasako M, Kinoshita T, et al. Can sentinel node biopsy indicate rational extent of lymphadenectomy in gastric cancer surgery? Fundamental and new information on lymph-node dissection. Langenbecks Arch Surg 1999; 384:149-57.

28. Miyashiro I, Hiratsuka M, Sasako M, et al. High falsenegative proportion of intraoperative histological examination as a serious problem for clinical application of sentinel node biopsy for early gastric cancer: final results of the Japan Clinical Oncology Group multicenter trial JCOG0302. Gastric Cancer 2014;17:316-23.

29. Kitagawa Y, Takeuchi H, Takagi Y, et al. Sentinel node mapping for gastric cancer: a prospective multicenter trial in Japan. J Clin Oncol 2013;31:3704-10.

30. Clarke D, Newcombe RG, Mansel RE. The learning curve in sentinel node biopsy: the ALMANAC experience. Ann Surg Oncol 2004;11:211S-5S.

31. Kinami S, Oonishi T, Fujita J, et al. Optimal settings and accuracy of indocyanine green fluorescence imaging for sentinel node biopsy in early gastric cancer. Oncol Lett 2016;11:4055-62.

32. Wang Z, Dong ZY, Chen JQ, et al. Diagnostic value of sentinel lymph node biopsy in gastric cancer: a metaanalysis. Ann Surg Oncol 2012;19: 1541-50.

33. Hayashi H, Ochiai T, Mori M, et al. Sentinel lymph node mapping for gastric cancer using a dual procedure with dye- and gamma probe-guided techniques J Am Coll Surg 2003;196:68-74.

34. Takeuchi H, Ueda M, Oyama T, et al. Molecular diagnosis and translymphatic chemotherapy targeting sentinel lymph nodes of patients with early gastrointestinal cancers. Digestion 2010; 82:187-91.
35. Yaguchi Y, Sugasawa H, Tsujimoto H, et al. One-step nucleic acid amplification (OSNA) for the application of sentinel node concept in gastric cancer. Ann Surg Oncol 2011;18:2289-96.

36. Kusano M, Tajima Y, Yamazaki K, et al. Sentinel node mapping guided by indocyanine green fluorescence imaging: a new method for sentinel node navigation surgery in gastrointestinal cancer Dig Surg 2008;25:103-8.

37. Miyashiro I, Miyoshi N, Hiratsuka M, et al. Detection of sentinel node in gastric cancer surgery by indocyanine green fluorescence imaging: comparison with infrared imaging Ann Surg Oncol 2008;15:1640-3.

38. Tajima Y, Yamazaki K, Masuda Y, et al. Sentinel node mapping guided by indocyanine green fluorescence imaging in gastric cancer Ann Surg 2009;249:58-62.

39. Toyota T, Fujito H, Suganami A, et al. Near-infraredfluorescence imaging of lymph nodes by using liposomally formulated indocyanine green derivatives. Bioorg Med Chem 2014;22:721-7.

40. Brouwer OR, Buckle T, Vermeeren L, et al. Comparing the hybrid fluorescent-radioactive tracer indocyanine green-99mTc-nanocolloid with 99mTc-nanocolloid for sentinel node identification: a validation study using lymphoscintigraphy and SPECT/CT. J Nucl Med 2012;53:1034-40.

41. Kiyozaki H, Saito M, Chiba H, et al. Laparoscopic wedge resection of the stomach for gastrointestinal stromal tumor (GIST): non-touch lesion lifting method. Gastric Cancer 2014;17:337-40.

42. Yang F, Wang S, Sun S, et al. Factors associated with endoscopic full-thickness resection of gastric submucosal tumors. Surg Endosc 2015;29:3588-93.

43. Hiki N, Yamamoto Y, Fukunaga T, et al. Laparoscopic and endoscopic cooperative surgery for gastrointestinal stromal tumor dissection. Surg Endosc 2008;22:1729-35.

44. Morita A, Yabushita K, Kimura J, et al. A case of stage IB gastric cancer caused peritoneal dissemination in 1 year after perforation during endoscopic submucosal dissection. Jpn J Gastroenterol Surg 2007;40:399-404.

45. Nunobe S, Hiki N, Gotoda T, et al. Successful application of laparoscopic and endoscopic cooperative surgery (LECS) for a lateral-spreading mucosal gastric cancer. Gastric Cancer 2012;15:338-42.

46. Inoue $H$, Ikeda $H$, Hosoya $T$, et al. Endoscopic mucosal resection, endoscopic submucosal dissection, and beyond: full-layer resection for gastric cancer with nonexposure technique (CLEAN-NET). Surg Oncol Clin N Am 2012;21:129-40. 
47. Goto O, Mitsui T, Fujishiro M, et al. New method of endoscopic full-thickness resection: a pilot study of nonexposed endoscopic wall-inversion surgery in an ex vivo porcine model. Gastric Cancer 2011;14:183-7.

doi: $10.21037 / \operatorname{tgh} .2017 .05 .02$

Cite this article as: Kinami S, Kosaka T. Laparoscopic sentinel node navigation surgery for early gastric cancer. Transl Gastroenterol Hepatol 2017;2:42.
48. Kitakata S, Itoh T, Kinami S, et al. Sentinel node navigated sealed EFTR for early gastric cancer. Operation 2015;69:1465-71. 